\title{
Local-field enhancement in an optical force metallic nanotrap: application to single-molecule spectroscopy
}

\author{
Patrick C. Chaumet, Adel Rahmani, and Manuel Nieto-Vesperinas
}

\begin{abstract}
We study the local-field enhancement in a nanocavity created by optical nanomanipulation. Recently we showed that a metallic probe can modify the optical force experienced by a metallic particle and generate a material selective trapping potential. We show that the same configuration used for optical forces can be used to control both in magnitude and tune the local-field enhancement around the particle at resonance. The spatial resolution and material selectivity of this technique, allied to its capability to manipulate particles at the nanometric level, may offer a new and versatile way to achieve surface-enhanced Raman scattering spectroscopy at the single-molecule level. @ 2006 Optical Society of America
\end{abstract}

OCIS codes: $260.2110,300.0300,290.0290$.

\section{Introduction}

The large enhancement of electromagnetic field intensities near metallic nanostructures can be used to magnify the intrinsic optical signature of physical and biological systems. In particular, the notoriously weak cross section of Raman scattering processes can be dramatically increased in the vicinity of metallic particles. The extreme sensitivity of surface-enhanced Raman scattering (SERS) spectroscopy has fostered many exciting applications, from biology and biochemistry to neurochemistry and DNA sequencing (see Ref. 1 for a review). However, one of the most challenging goals in SERS is to achieve the sensitivity required for the fast detection and identification of single molecules. The ability to select a given metallic particle among several with different shapes and nature would allow one to single out the particle exhibiting the best electromagnetic enhancement at the required frequency, thus offering a significant asset for the study of single molecules.

P. C. Chaumet (patrick.chaumet@fresnel.fr) is with the Institut Fresnel (UMR 6133), Université Paul Cézanne, Avenue Escadrille Normandie-Nieman, F-13397 Marseille Cedex 20, France. A. Rahmani is with the Laboratoire d'Electronique, Optoélectronique et Microsystèmes-UMR CNRS 5512-Ecole Centrale de Lyon 36, Avenue Guy de Collongue, F-69134 Ecully Cedex, France. M. NietoVesperinas is with the Instituto de Ciencia de Materiales de Madrid, Consejo Superior de Investigaciones Cientificas, Campus de Cantoblanco, Madrid 28049, Spain.

Received 7 October 2005; revised 13 January 2006; accepted 26 January 2006; posted 6 February 2006 (Doc. ID 65247).

$0003-6935 / 06 / 215185-06 \$ 15.00 / 0$

(C) 2006 Optical Society of America
Optical near-field microscopy has become a common tool for optical nanocharacterization (for a review on near-field optical imaging techniques see Ref. 2 ). Among the different techniques, apertureless microscopy exploits the highly localized strong field enhancement near the apex of a metallic probe. ${ }^{3-5}$ This enhancement is due to the excitation of plasmon modes and depends on the shape and the nature of the tip. ${ }^{6}$ Moreover, the presence of a particle close to the tip can further enhance the field amplitude: for instance, a $10 \mathrm{~nm}$ particle near a metallic probe can provide adequate field enhancement for SERS experiments. ${ }^{6}$ Also when the tip is close to a metallic substrate it is well known that the system can support a tip-induced surface plasmon mode and that the resonance depends on the distance between the tip and the surface, the shape of the tip, and the nature of both tip and surface. ${ }^{7-9}$ Even in the case of a dielectric substrate, as the tips gets closer the substrate the field enhancement increases. ${ }^{10,11}$

Recently, we proposed a method that uses optical forces to selectively manipulate dielectric particles above a dielectric substrate. ${ }^{12}$ The selective manipulation is achieved by using a tungsten apertureless near-field probe to shape an evanescent field into a three-dimensional optical trap that can be used to capture and manipulate dielectric particles. ${ }^{13}$ Further, we showed that in the case of metallic particles the optical trapping can be tailored to be material selective, thus allowing one to create and control metallic nanocavities. ${ }^{14}$ As shown in Ref. 4, the ability to engineer metallic nanocavities would be an important asset for the control of SERS processes at the molecular level. Thus our configuration could allow 


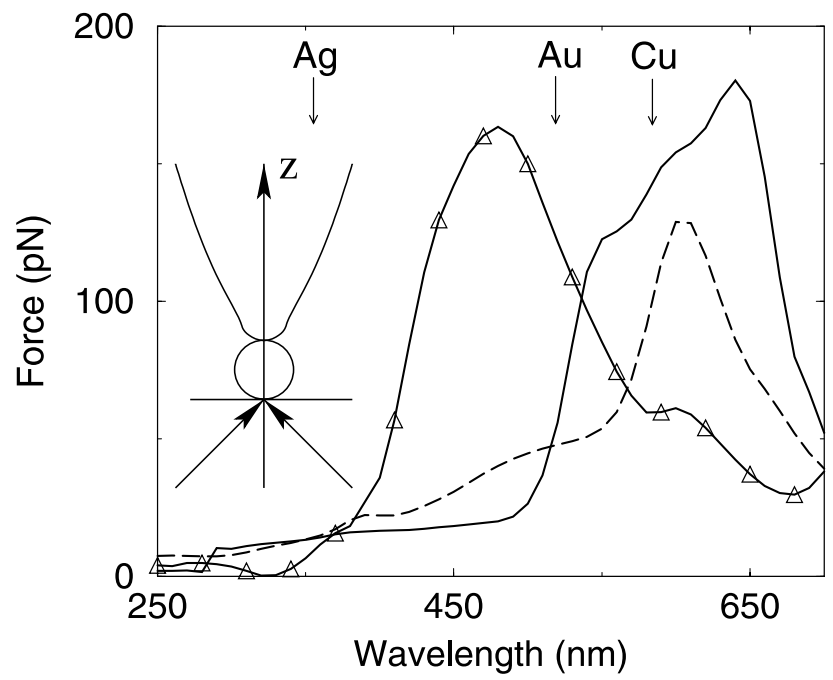

Fig. 1. $z$ component of the optical force experienced by a sphere of radius $a=10 \mathrm{~nm}$ versus the wavelength of illumination. The tungsten tip is in contact with the sphere. Solid curve, gold sphere; dashed curve, copper sphere; triangle, silver sphere. The arrows indicate the plasmon resonance wavelengths of an isolated sphere.

one to study SERS for a variety of nanocavity geometries and nature within a single experiment by exploiting optical forces. In this paper we explore the potential of the nanocavities created by nanomanipulation for creating local-field enhancements between a near-field probe and a particle on a substrate. Note that it is of great interest to study such nanocavities. First because it allows for selective nanomanipulation, and second because it produces a highly localized field enhancement, for the configuration we consider that the enhancement of the field is localized laterally at approximately $10 \mathrm{~nm}$. In Section 2 we compute the optical force experienced by a sphere in gold, silver, and copper versus the wavelength in the presence of a tungsten tip. In Section 3 we address the SERS process. We study the influence on the SERS factor of the wavelength, the nature of the cavity (the material of the probe and the particle), and its geometry (the distance between the probe and the sphere). In Section 4 we briefly illustrate the quantum electrodynamic effects such as the modification of spontaneous emission in such nanocavities. We present our conclusions in Section 5.

\section{Principle of Optical Nanomanipulation}

Since the optical nanomanipulation on metallic spheres has been done previously, we will very briefly restate the principle. ${ }^{14}$ We consider a metallic particle in air (or vacuum) above a dielectric substrate (permittivity $\epsilon=2.25$ ). The particle is illuminated by two counterpropagating evanescent waves with the same polarization and a random phase relation. ${ }^{13}$ These evanescent waves create a vertical gradient optical force. By using a metallic probe, this force can be shaped to produce a three-dimensional optical trap. Figure 1 shows the $z$ (i.e., vertical) component of the force experienced by a metallic sphere (gold, sil- ver, or copper) with radius $a=10 \mathrm{~nm}$ lying on the flat dielectric substrate, versus the illumination wavelength. The tungsten tip is above the sphere and in contact with it. ${ }^{13}$ The tip has a parabolic shape, but note that the angle at the tip apex is not a critical limiting factor for achieving high field enhancement. ${ }^{6}$ The radius at the apex of the tip is $10 \mathrm{~nm}$. To avoid a tedious computation while getting the effect of an extended tip, we truncate the tip at more than a wavelength, i.e., at $800 \mathrm{~nm}$. Note that the spacing lattice used for the discretization is less than $2 \mathrm{~nm}$ for the spheres and at the apex of the tip, areas where the variation of the field is most rapid. Away from the apex of the tip the lattice discretization is coarser. With this discretization, the spherical particle to trap with a diameter of $20 \mathrm{~nm}$ is discretized in 739 subunits. Only the TM polarization leads to a positive force and is shown thus in Fig. 1. The complex permittivities of metals are taken from Ref. 15 . Note that the computation of the optical forces is done in two stages. First, the field inside the objects (in our case it is the particle on the substrate and the tip) is obtained through the coupled dipole method (CDM). ${ }^{16}$ Second, the optical forces are computed using the Maxwell stress tensor technique. The details on the theory can be found in Ref. 17 . Note that once the field inside the objects is obtained, the CDM can also be used to compute the diffracted field at any position outside the objects under study. ${ }^{16}$

For the three metallic spheres of Fig. 1 the force strongly depends on the wavelength of illumination. Hence it is possible to perform a selective nanomanipulation. For example, at $\lambda=325 \mathrm{~nm}$ the force experienced by the silver sphere vanishes, and this sphere cannot be manipulated, whereas at $\lambda=530 \mathrm{~nm}$ the optical force is 2.5 times larger for the gold sphere than for the copper sphere. Note that the resonance is a plasmon resonance for gold and silver whereas it corresponds to an interband transition for copper.

\section{Local-Field Enhancement Tuning: Application to Surface-Enhanced Raman Scattering}

The ability to selectively manipulate small metallic particles can be exploited for spectroscopic studies of biological systems. It is possible to use the field enhancement created by the tip alone to do Raman spectroscopy, ${ }^{18}$ however, the use of particles to engineer nanocavities can offer a greater versatility. Let us consider the configuration in which a molecule is adsorbed on a sphere. Neglecting the Stokes shift, the electromagnetic enhancement factor relevant to SERS is expressed as $M=\left(E / E_{0}\right)^{4}$, where $E$ is the electric field at the position of the molecule and $E_{0}$ is the magnitude of the electric field of the plane wave that undergoes total internal reflection at the substrate-air interface (the SERS study and the trapping can be performed at two different wavelengths). We note that it was recently suggested that the strong optical force near metallic particles could be used to trap molecules in a liquid environment. 19,20 In the configuration addressed in this paper, the trap- 

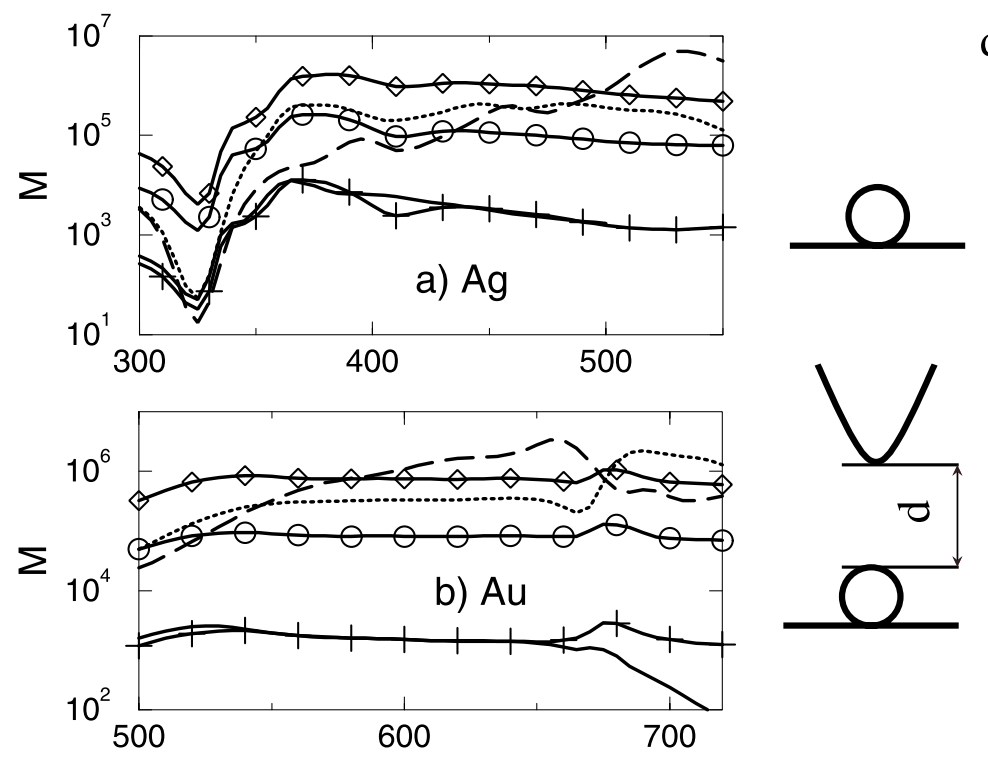

d)
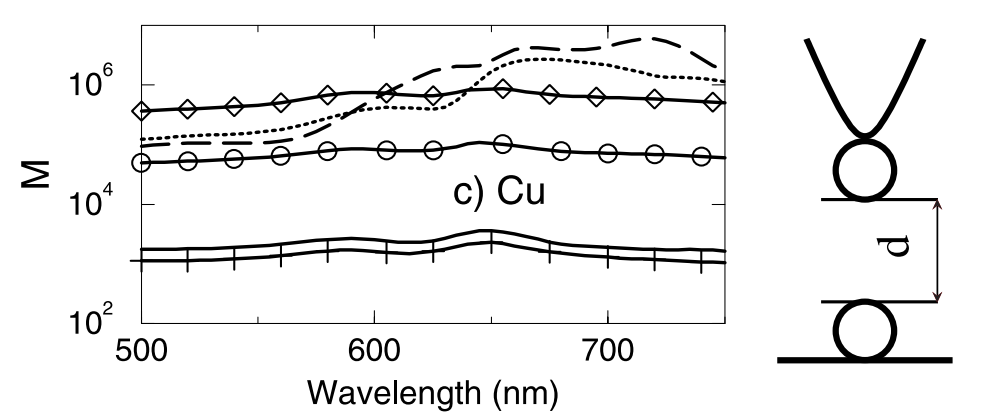

Solid curve

crosses $\quad \mathrm{d}=30 \mathrm{~nm}$

circles $\quad \mathrm{d}=8 \mathrm{~nm}$

diamonds $\quad \mathrm{d}=4 \mathrm{~nm}$

dashed curve $\mathrm{d}=4 \mathrm{~nm}$

dotted curve $\mathrm{d}=4 \mathrm{~nm}$

Fig. 2. SERS electromagnetic enhancement factor $(M)$ versus the wavelength of illumination for a molecule located $2 \mathrm{~nm}$ from the sphere, for different distances $d$ between the tungsten tip and the sphere: $d=\infty$ (solid curves), $d=30 \mathrm{~nm}$ (crosses), $d=8 \mathrm{~nm}$ (circles), and $d=$ $4 \mathrm{~nm}$ (diamonds). Dashed curves pertain to cases in which the tip is of the same material as the sphere and $d=4 \mathrm{~nm}$. Dotted curves pertain to cases in which the tip is in tungsten, with a second sphere (same material as the first one) trapped at its apex. Distance between the two spheres $d=4 \mathrm{~nm}$. The radius of the spheres is $10 \mathrm{~nm}$. (a) Silver sphere, (b) gold sphere, (c) copper sphere.

ping of a molecule between the tip and the sphere in a liquid environment is possible. However, the particle size and nature and the temperature must be such that Brownian motion does not upset the selection and trapping of the metallic particle by the tip. Also the magnitude of the intensity between the tip and the particle must be such that the molecule stays trapped between both in spite of the Brownian motion. According to the calculations presented in Ref. 19 , in a liquid environment, a tip-particle separation on the order of $1 \mathrm{~nm}$ would therefore be required. Figure 2 shows the enhancement factor $M$ for a molecule adsorbed on the metallic sphere for different distances between the sphere and the tip. We assume that the molecule is $2 \mathrm{~nm}$ away from the surface of the sphere and located underneath the tip.

We see that when the tip is not present (the solid curves in Fig. 2) the maximum enhancement is obtained near the plasmon resonance of the sphere, the substrate being responsible for a slight redshift of the resonance. This maximum is at $365 \mathrm{~nm}$ for silver, $525 \mathrm{~nm}$ for gold, and $650 \mathrm{~nm}$ for copper (the solid curve in Fig. 2). When the tip is $30 \mathrm{~nm}$ away from the sphere (the dotted curve in Fig. 2) the tip-sphere interaction is too weak to entail significant changes in the magnitude of the field enhancement, except for gold where above $\lambda=670 \mathrm{~nm}$ the curve departs from that of an isolated sphere. Compared to the case without a tip, the position of the resonance is redshifted by approximately $5 \mathrm{~nm}$ for the silver and copper spheres and approximately $140 \mathrm{~nm}$ for the gold sphere. This large shift for the gold sphere comes from a slow decrease of the real part of the permittivity of gold between 500 and $700 \mathrm{~nm}$ and a minimum of the imaginary part at $\lambda=650 \mathrm{~nm}$. As the tip gets closer to the sphere, the redshift of the resonance peak for the silver sphere becomes more noticeable (reaching approximately $20 \mathrm{~nm}$ for a tip $4 \mathrm{~nm}$ away from the sphere compared to an isolated sphere). Conversely, for the copper sphere, the position of the resonance peak remains close to $650 \mathrm{~nm}$. In general, approaching the tip near the sphere creates a redshift of the resonance. This effect has also been observed with two spheres. ${ }^{21}$ For the three different metallic spheres the local-field enhancement increases as the tip approaches the particle, yielding a SERS factor of the order of $10^{6}$ when the tip is only $4 \mathrm{~nm}$ away from the 
sphere. Note that this factor accounts only for the electromagnetic part of SERS. ${ }^{22}$ For the sake of computation time, we did not compute $M$ for smaller distances between the tip and the sphere. But for smaller separations, in view of the results of Refs. 4, 10 , and 22 the enhancement factor would be even larger. Since the plasmon-mode resonance depends on the size of the cavity formed by the tip and the sphere, we have changed the cavity width by varying the distance between the tip and the sphere. However, the geometry of the cavity, and hence the SERS factor, would also change with the radius of curvature of the tip and/or the particle. Changing the material of the tip will also modify both the position of the plasmon resonance and the factor $M$ as is demonstrateld in Fig. 2 [the dashed curve in Fig. 2(a): silver tip; the dashed curve in Fig. 2(b): gold tip; the dashed curve in Fig. 2(c): copper tip; tip-particle distance: $4 \mathrm{~nm}$ ]. We see that for a given geometry the maximum enhancement is largest when the tip is of the same material as the sphere (a metal that presents plasmon resonance) instead of a tungsten tip. As expected, the spectral location of the resonance also depends strongly on the nature of the tip. When the tip is near the particle we observe a shift of $30 \mathrm{~nm}$ for a gold tip, $200 \mathrm{~nm}$ for a silver tip, and $70 \mathrm{~nm}$ for a copper tip, compared to those for a tungsten tip. A simplified model can be used to further illustrate the influence of the different parameters on the spectral position of the plasmon mode of the cavity. Let us assume that the optical properties of both the tip and the particle can be described by a Drude model for two spheres made of different metals with a bulk plasmon resonance at $\omega_{1}$ and $\omega_{2}$, respectively. When the distance $d$ between the surface of the two spheres is smaller than their radii $\left(R_{1}\right.$ and $\left.R_{2}\right)$, a simple calculation, as in Ref. 23, shows that the frequency of the plasmon mode of the cavity is

$$
\omega=\frac{\omega_{1} \omega_{2} \sqrt{\pi}}{\sqrt{\omega_{1}^{2}+\omega_{2}^{2}}}\left[\frac{\left(R_{1}+R_{2}\right) d}{8 R_{1} R_{2}}\right]^{1 / 4} .
$$

Note that this is the fundamental frequency of a set of discrete cavity resonances. This expression for the frequency of the two-particle resonance is valid owing to the size of the spheres considered here and to the fact that the dipolar mode is the strongest. ${ }^{24}$ This expression shows that the resonance is redshifted when $d$ decreases. However, from Eq. (1) we can also see that, for a given distance $d$ between the tip and the sphere, the redshift of the resonance will also depend on the size of the particle $\left(R_{2}\right)^{25}$ Although resulting from a simple model, Eq. (1) illustrates the fact that several parameters can be used to control, to some extent, the spectral position of the resonance and the magnitude of the enhancement factor $M$, for instance, by varying the distance between the tip and the particle, or by selecting a particle of a different size. Another advantage is that it creates a highly localized electromagnetic enhancement as the plas- mon mode of the cavity is confined within a region a few nanometers in size (approximately $6-7 \mathrm{~nm}$ in our case) underneath the tip. Therefore the tip provides the spatial selectivity necessary to restrict the SERS process to a single molecule.

Furthermore, one can also use a given tip to manipulate one particle over another where a molecule would be adsorbed. This would create a cavity whose properties can be modified by changing the trapped particle. To illustrate this, we plotted in Fig. 2 (the dotted curves) the enhancement for a cavity made of two spheres of the same metal. One sphere lies on the substrate while the other is trapped by a tungsten tip and placed $4 \mathrm{~nm}$ above the first sphere. We emphasized the stability of this system, i.e., the second particle remained trapped by the tip when they both approached the first particle. Irrespective of the nature of the particle, the magnitude of the electromagnetic enhancement remains of the same order of magnitude as the one achieved with the tip above a single sphere. Note that for the gold and copper spheres the resonance is redshifted compared to the case of a single sphere. Incidentally, we note that in this configuration the optical force tends to bring the spheres together, which would increase $M$. If we compare the factor $M$ of this configuration to the one obtained between two gold spheres (radius $a=$ $10 \mathrm{~nm}$ ) separated by $4 \mathrm{~nm}$ in free space, we find that our SERS enhancement factor is approximately 1000 times larger. We emphasize that this large enhancement is due to the illumination by evanescent waves, although the angle of incidence is close to the critical angle $\left(\theta=43^{\circ}\right)$, and hence the evanescent waves are slowly decaying. Indeed, if we illuminate the system with a plane wave from above the substrate, we obtain a factor $M$ weaker by at least a factor of 10 . Note that the manipulation and the SERS study can be performed at different wavelengths to avoid molecular bleaching.

At first glance it may seem that our enhancement factor $M$ is smaller than those usually found in the literature (often approximately $10^{12}$ ). In fact, our configuration is actually very efficient, and if $M$ seems smaller it is due to two things. The first one is the distance between the two objects, which is $4 \mathrm{~nm}$ for the sake of computation time and memory [to achieve convergence, the field should be computed at least one spacing lattice away from the objects (tip or sphere), therefore a smaller distance between the tip and the sphere requires a finer discretization]. In the literature, particles are often as close as $1 \mathrm{~nm}$. As the field between two sphere varies more or less as $1 / d^{2}, 26$ this accounts for two orders of magnitude. The second one is more subtle and relates to the size of the spheres we use. The radius (tip and sphere) is $10 \mathrm{~nm}$, and as the field scattered by a sphere is proportional to the cube of its radius, the factor $M$ is smaller than for larger spheres. For example, for two gold spheres, with a radius of $10 \mathrm{~nm}$ and distance between them of $5.5 \mathrm{~nm}, \mathrm{Xu}$ et $a l .{ }^{22}$ find $M=10^{3}$ (we have done the computation with our method for the spheres alone, 


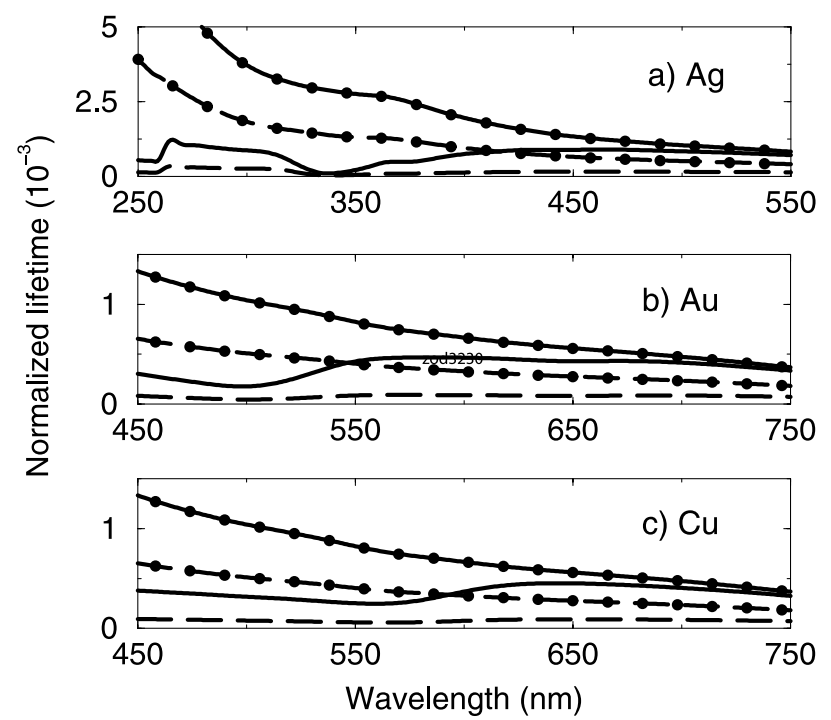

Fig. 3. Lifetime normalized to free space for dipoles parallel (solid curve) and perpendicular (dashed curve) to the wall of a plane junction used to model the tip-sphere cavity for (a) silver, (b) gold, and (c) copper. Distance between the tip in tungsten and the sphere $d=4 \mathrm{~nm}$ (solid curves without symbol) and $d=30 \mathrm{~nm}$ (curves with circles).

and we find the same factor $M$ for that configuration). For the same spheres and $d=4 \mathrm{~nm}$, in our configuration, we find $M$ to be approximately $10^{7}$. Hence our configuration is actually more efficient at achieving large enhancement with small particles.

Note that we trap the particles in TM polarization, which is the polarization used for the SERS experiments. There is no obvious correlation between the optical force experienced by the particle and the enhancement of the field between the tip and the particle. This can be see in the fact that the resonances of the two phenomena do not occur exactly at the same wavelength. We think that this is due to the fact that the optical force (mainly due to the gradient force) depends on the derivative of the local field, which is not necessary maximum when the field is maximum; but it should be interesting to use the same illumination for both the optical trap and the SERS experiment. For example, for a gold particle and a tungsten tip the maximum for the SERS enhancement factor is approximately $\lambda=570 \mathrm{~nm}$ where the value of optical force is still approximately $50 \mathrm{pN}$. At that wavelength the particle is attracted by the tip, and the effect of the optical force is to create a trap with a small gap and therefore a large SERS enhancement factor.

\section{Spontaneous Emission Modification}

The large electromagnetic enhancement found for SERS suggests that the present configuration may also be useful for quantum electrodynamic studies. Indeed the subwavelength cavity created between the tip and the particle (or between two particles) can significantly alter the dynamics of a nanosource. Figure 3 shows the lifetime of an atomic dipole transition, normalized to that in free space. For the sake of computational simplicity, the calculation of the spontaneous emission lifetime was done for a three medium junction, ${ }^{27}$ i.e., the tip and the sphere are both assumed to be semi-infinite media limited by a plane interface with air. The tip-sphere distance is now represented by the distance between the two surfaces. This is an acceptable model, as preliminary calculations showed that the three medium junction configuration yielded the correct order of magnitude for the spontaneous emission rates in the tip-particle cavity. This is due to the fact that the spontaneous emission process involves the electromagnetic local density of states near the metal whose main contributions, away from the surface plasmon resonance of a planar surface, are the longitudinal field modes that are localized. In Fig. 3 the molecule is $2 \mathrm{~nm}$ from the sphere, and the distance between the tip and the sphere is $d=4 \mathrm{~nm}$ (the curves without symbols) and $d=30 \mathrm{~nm}$ (the curves with circles). When the tip approaches the sphere its effect is to reduce the lifetime of the molecule, for both orientations of the dipole, due to evanescent mode frustration, absorption, and coupling to surface plasmon. This is particularly noticeable for silver; the lifetime becomes very small at $\lambda=340 \mathrm{~nm}$, which corresponds to the surface plasmon resonance.

Note that, when the dipole is perpendicular to the substrate, the maximum of the normalized lifetime is obtained close to the maximum of $M$ for the same configuration (the tip in tungsten above a spherical particle).

\section{Conclusion}

In summary, we proposed to use the enhancement of optical forces at the apex of a metallic probe to perform the selective trapping and manipulation of nanoparticles with intrinsic spectroscopic signatures. By using (weakly) evanescent illumination we were able to obtain local-field enhancements larger than those found with plane wave illumination. Although we considered metallic particles, the principle of our scheme can be extended to any nanoscopic system exhibiting intrinsic resonances. ${ }^{28}$ Since it allows the identification and the selection of resonant nanoparticles, this approach can be used to achieve high SERS enhancement factors at the single-molecule level. As our calculations show, by moving the tip from one particle to another, one can change the properties of the microcavity and therefore control the magnitude and spectrally tune the electromagnetic enhancement.

A. Rahmani thanks the Ministère délégué à la Recherche et aux Nouvelles Technologies for funding (ACI Jeune Chercheur).

\section{References}

1. K. Kneipp, H. Kneipp, I. Itzkan, R. R. Dasari, and M. S. Feld, "Surface-enhanced Raman scattering and biophysics," J. Phys.: Condens. Matter 14, R597-R624 (2002).

2. G. P. Wiederrecht, "Near-field optical imaging of noble metal nanoparticles," Eur. Phys. J.: Appl. Phys. 28, 3-18 (2004).

3. L. Novotny, R. X. Bian, and X. S. Xie, Phys. Rev. Lett. 79, 645-648 (1997). 
4. M. Micic, N. Klymyshyn, Y. D. Suh, and H. P. Lu, "Finite element method simulation of the field distribution for AFM tip-enhanced surface-enhanced Raman scanning microscopy," J. Phys. Chem. B 107, 1574-1584 (2003).

5. Y. C. Martin, H. F. Hamann, and H. K. Wickramasinghe, "Strength of the electric field in apertureless near-field optical microscopy," J. Appl. Phys. 89, 5774-5778 (2001).

6. J. T. Krug, E. J. Sánchez, and X. S. Xie, "Design of near-field optical probes with optimal field enhancement by finite difference time domain electromagnetic simulation," J. Chem. Phys. 116, 10895-10901 (2002).

7. J. A. Porto, P. Johansson, S. P. Apell, and T. López-Ríos, "Resonance shift effects in apertureless scanning near-field optical microscopy," Phys. Rev. B 67, 085409 (2003).

8. R. Berndt, J. K. Gimzewski, and P. Johansson, "Inelastic tunneling excitation of tip-induced plasmon modes on noble-metal surfaces," Phys. Rev. Lett. 67, 3796-3799 (1991).

9. R. Berndt, J. K. Gimzewski, and P. Johansson, "Electromagnetic interactions of metallic objects in nanometer proximity," Phys. Rev. Lett. 71, 3493-3496 (1993).

10. R. G. Milner and D. Rochards, "The role of tip plasmons in near-field Raman microscopy,” J. Microsc. 202, 66-71 (2001).

11. F. Festy, A. Demming, and D. Richards, "Resonant excitation of tip plasmons for tip-enhanced Raman SNOM," Ultramicroscopy 100, 437-441 (2004).

12. P. C. Chaumet, A. Rahmani, and M. Nieto-Vesperinas, "Optical trapping and manipulation of nano-objects with an apertureless probe," Phys. Rev. Lett. 88, 123601-123605 (2002).

13. P. C. Chaumet, A. Rahmani, and M. Nieto-Vesperinas, "Selective nanomanipulation using optical forces," Phys. Rev. B 66, 195405 (2002).

14. P. C. Chaumet, A. Rahmani, and M. Nieto-Vesperinas, "Photonic force spectroscopy on metallic and absorbing nanoparticles," Phys. Rev. B 71, 045425 (2005).

15. E. D. Palik ed., Handbook of Optical Constants of Solids (Academic, 1985).

16. P. C. Chaumet, A. Rahmani, F. de Fornel, and J.-P. Dufour, "Evanescent light scattering: The validity of the dipole approximation," Phys. Rev. B 58, 2310-2315 (1998).

17. P. C. Chaumet and M. Nieto-Vesperinas, "Coupled dipole method determination of the electromagnetic force on a particle over a flat dielectric substrate," Phys. Rev. B. 61, 1411914127 (2000).

18. A. Hartschuh, E. J. Sánchez, X. S. Xie, and L. Novotny, "Highresolution near-field raman microscopy of single-walled carbon nanotubes," Phys. Rev. Lett. 90, 095503 (2003).

19. H. Xu and M. Käll, "Surface-plasmon-enhanced forces in silver nanoaggregates," Phys. Rev. Lett. 89, 246802 (2002).

20. N. Calander and M. Willander, "Optical trapping of single molecules at the detection spots of nanoprobes," Phys. Rev. Lett. 89, 143603-143608 (2002).

21. J. M. Gérardy and M. Ausloos, "Absorbtion spectrum of clusters of spheres from the general solution of Maxwell's equation: II. Optical properties of aggregated metal spheres," Phys. Rev. B 30, 2167-2181 (1984).

22. H. Xu, J. Aizpurua, M. Käll, and P. Apell, "Electromagnetic contributions to single-molecule sensitivity in surface-enhanced Raman scattering," Phys. Rev. E 62, 4318-4324 (2000).

23. R. W. Rendell and D. J. Scalapino, "Surface plasmons confined by microstructures on tunnel junctions," Phys. Rev. B 24, 3276-3294 (1981).

24. A. A. Lushnikov, V. V. Maksimenko, and A. J. Simonov, in Electromagnetic Surface Modes, A. D. Boardman, ed. (Wiley, 1982), p. 305.

25. P. Johansson, "Light emission from a scanning tunneling microscope: fully retarded calculation," Phys. Rev. B 58, 1082310834 (1998).

26. We have checked this dependence in the static case (because the system of two spheres is smaller than the wavelength of the illumination) by performing the computation in the bispherical coordinates. [P. C. Chaumet and J.-P. Dufour, "Electric potential and field between two different spheres," J. Electrost. 43, 145-149 (1998)].

27. A. Rahmani, P. C. Chaumet, and F. de Fornel, "Environmentinduced modification of spontaneous emission: single-molecule near-field probe," Phys. Rev. A 63, 023819 (2001).

28. T. Iida and H. Ishihara, "Theoretical study of the optical manipulation of semiconductor nanoparticles under an excitonic resonance condition," Phys. Rev. Lett. 90, 057403 (2003). 